Check for updates

Cite this: RSC Adv., 2018, 8, 36114

Received 28th July 2018

Accepted 18th October 2018

DOI: $10.1039 / c 8 r a 06355 a$

rsc.li/rsc-advances

\section{Construction of sandwich-structured CoAl-layered double hydroxide azeolitic imidazolate framework- 67 (CoAl-LDH@ZIF-67) hybrids: towards enhancing the fire safety of epoxy resins}

\begin{abstract}
Hailin Guo, ${ }^{a}$ Yifan Wang, ${ }^{a}$ Chaofeng Li ${ }^{\mathrm{a}}$ and Keqing Zhou (D) *ab
In this work, sandwich-like structured CoAl-LDH@ZIF-67 hybrids were constructed by in situ growth of nanosized ZIF-67 crystallites on CoAl-LDH nanoplates. The structure and morphology of CoAlLDH@ZIF-67 hybrids were represented by X-ray diffraction (XRD), Fourier Transform Infrared Spectroscopy (FTIR) and Transmission Electron Microscopy (TEM). Accordingly, the hybrids were introduced into epoxy resins to explore their effect on the thermal and fire safety properties of epoxy composites. With the introduction of $2 \%$ CoAl-LDH@ZIF-67 hybrids, the $T_{10 \%}, T_{50 \%}$ and $T_{\max }$ values were decreased to some extent and the char residue yield was higher than that of pristine epoxy. The peak heat release rate (PHRR) of epoxy composites declined by $26.4 \%$, in comparison with those of pure epoxy. Moreover, the quantity of smoke produced, toxic $\mathrm{CO}$ released and $\mathrm{CO}_{2}$ produced were suppressed markedly and decreased by $16.7 \%, 59.8 \%$ and $32.2 \%$, respectively. A possible mechanism for improving fire safety was put forward based on the analysis of the carbon residues.
\end{abstract}

\section{Introduction}

EP is a class of polymers containing two or more epoxy groups in the molecule, with excellent chemical and mechanical properties. It plays an important role in coatings, adhesives, laminates and electronics. ${ }^{1-3}$ However, the flammability of EP, producing plumes of smoke and toxic gas in the process of burning, excludes EP from further applications. Many methods are posed to solve this problem. Generally, adding additives like fillers, diluters and specific resins is an efficient approach to intensify the performances of EP. Therefore, exploring the agents which can be applied to enhance the flame retardancy and smoke suppression performances of EP is a research hotspot.

A large amount of nanofillers have been developed to raise the fire resistance of EP composites. Among those, layered double hydroxides (LDH), mixed metal hydroxides of two kinds of metal ions with a layered structure, have attracted considerable research interest, because $\mathrm{LDH}$ can be used as an additive in polymer composites to enhance heat stability and fire safety of the entire system. Three mainly flame retardant and smoke suppression mechanisms are generally accepted. Firstly, the thermal degradation and vaporization process of water in the

${ }^{a}$ Faculty of Engineering, China University of Geosciences, Wuhan, Hubei, 430074, PR China.E-mail: zhoukq@cug.edu.cn

${ }^{b}$ Engineering Research Center of Rock-Soil Drilling \& Excavation and Protection, China University of Geosciences (Wuhan), Ministry of Education, Wuhan, Hubei, 430074, PR China structure are all endothermic and can reduce temperature of the system. ${ }^{4-7}$ Secondly, the catalytic effects of pyrolysis products play an important role. According to previous work, ${ }^{8}$ these characteristics, rooted in their brucite-like chemical structure, arouse an endothermic decomposition reaction involving the production of metal oxides. Notably, these metal oxides have certain catalytic effect in thermal reaction. Thirdly, the structure of residues would slow down subsequent burning by reducing the oxygen supply to the bulk phase under the burning surface. ${ }^{8}$ $\mathrm{Xu}$ and co-workers ${ }^{9}$ studied the flame retardancy and smoke suppression effect of EP composites laced with GO/LDH. Compared with pure EP, the PHRR and THR of EP composites were shorten by $31.1 \%$ and $7.0 \%$, respectively. Wang et al. prepared EP composites laced with $\mathrm{Ni}-\mathrm{Fe} \mathrm{LDH} /$ graphene hybrids to improve fire safety: when introducing $2.0 \mathrm{wt} \%$ of $\mathrm{LDH} /$ graphene mixtures, the PHRR and THR is obviously reduced by $38 \%$ and $48 \%$ in comparison with those of pure EP. ${ }^{10}$ Ehsan and co-workers concluded that SCD-DBS-T-LDH/EP composites exhibited better fire resistance than its counterparts under the same filler loading: the HRR, THR, TTI and FIGRA values of epoxy resins incorporating SCD-DBS-T-LDH vary greatly, relative to those of pristine EP. ${ }^{11}$

However, there are two defects of LDH as additive in polymer. One of the main barriers for the commercialization of $\mathrm{LDH}$ is the low utilization of $\mathrm{LDH}$, which means the high loading of LDH. ${ }^{12,13}$ On the other hand, interfacial interaction between LDH and polymer matrix is weak and it is difficult to obtain well dispersion state, which directly limits the application of LDH in 
polymers. ${ }^{14}$ However, it has been reported that the surface metal cations of LDH exist at an unsaturated coordination state..$^{15}$ This inspires researchers to refer to a crystalline material, metalorganic frameworks (MOFs), which are well-defined crystalline crystals with high surface and flexible structures. Such structure would result in strong attachment to polymer matrices and the organic linkers in MOFs can form firmer relation with polymer linkers. ${ }^{16}$ Meanwhile, MOFs are flame retardants for polymer materials to obtain excellent fire safety. Shi $e t$ al. found that the LOI value of PLA/ZIF-8 composites reached $26 \%$ by incorporation of $3 \mathrm{wt} \% \mathrm{ZIF}-8$, and its flame retardancy was improved. ${ }^{17} \mathrm{~A}$ cobalt-based metal-organic-framework containing phosphorus structure was prepared by Hou and co-workers. By loading of $2 \mathrm{wt} \%$ P-MOF, the PHRR and THR values of EP composites were reduced by $28 \%, 18.6 \%$, and significant reductions in TSP and total CO production were also observed, with $15 \%$ and $52 \%$ reductions, respectively. ${ }^{18}$

In previous work, Liu et al. have proved that LDH@MOF hybrids can be successfully prepared by in situ growth of nanosized MOF crystallites on LDH, the hexagonal nanoplate structure of $2 \mathrm{D} \mathrm{Zn}-\mathrm{Al} \mathrm{LDH}$ substances is maintained integrity after the introduction of ZIF-8, a branch of MOFs, and exhibit an excellent $\mathrm{CO}_{2}$ adsorption capacity. ${ }^{19} \mathrm{Li}$ et al. have designed sandwich structure by in situ nucleation on the surface of LDHs nanosheets and directed growth of MOFs on it. Subsequently, the heat treatment of above material is carried out to obtain a substance that has perfect electrocatalytic ORR performances. ${ }^{20}$ However, this structure is mainly focused on the electrical conductivity and catalytic performance at present, rarely on the thermal and fire safety performance of polymer materials.

Herein, sandwich-like structured CoAl-LDH@ZIF-67 hybrids were constructed by in situ growth of nanosized ZIF-67 crystallites on LDH. The structure and morphology of the CoAlLDH@ZIF-67 hybrids were illustrated and then used for enhancing the flame retardancy and smoke suppression performances of EP composites. The cone test results exhibit EP composites have good fire safety properties, which is crucial to develop new flame retardants and enrich polymer flame retardant systems.

\section{Experimental}

\subsection{Materials}

Cobalt nitrate hexahydrate, aluminum nitrate nonahydrate, urea, ethanol, methanol, acetone, 4,4-diaminodiphenyl methane were all provided from Shanghai Hushi Laboratorial Equipment Co., Ltd. 2-Methylimidazole (98\%) was supplied by Shanghai Aladdin Biochem Technology Co., Ltd. Epoxy resin (E44) was provided by Shandong Deyuan Epoxy Resin Co., Ltd.

\subsection{Preparation of CoAl-LDH}

CoAl-LDH with sheet-like shape was fabricated in a three-neck round-bottom flask provided with a condenser. ${ }^{21}$ Typically, $800 \mathrm{~mL}$ of ultrapure water was used to dissolved cobalt nitrate hexahydrate $(10 \mathrm{mmol})$, aluminum nitrate nonahydrate (5 $\mathrm{mmol}$ ) and urea (35 $\mathrm{mmol})$. Then, the resulting solution was heated and continuously stirred at $100{ }^{\circ} \mathrm{C}$ for $24 \mathrm{~h}$ and a reflux condenser that is passed through nitrogen works simultaneously. The final products were filtered, cleaned with deionized water and ethanol, and finally dried at $60^{\circ} \mathrm{C}$ for $6 \mathrm{~h}$.

\subsection{Preparation of sandwich-like structured CoAl-LDH@ZIF- 67 hybrids}

Sandwich-like structured CoAl-LDH@ZIF-67 hybrids were synthesized in methanol at room temperature. Typically, $0.1 \mathrm{~g}$ of CoAl-LDH was first dispersed in $50 \mathrm{~mL}$ of methanol, and then subjected to ultrasonic treatment for several hours and mechanically stirred to obtain a uniform suspension. $50 \mathrm{~mL}$ of cobalt nitrate hexahydrate methanol solution $(0.1 \mathrm{M})$ was added next. Then a 2-methylimidazole methanol solution $(50 \mathrm{~mL}, 0.8$ M) was dripped into the above solution under magnetic stirring at $25{ }^{\circ} \mathrm{C}$ for $15 \mathrm{~min}$. The products were obtained by centrifugation (3000 rpm, $5 \mathrm{~min}$ ), cleaned several times by methanol, and finally dried in an oven at $60{ }^{\circ} \mathrm{C}$ for $6 \mathrm{~h}$.

\subsection{Preparation of EP composites}

The preparation of EP composites containing $2.0 \mathrm{wt} \%$ CoAlLDH@ZIF-67 hybrids is explained as follows: $1.2 \mathrm{~g}$ of CoAlLDH@ZIF-67 hybrids were dispersed and continuous ultrasonic stirred in $50 \mathrm{~mL}$ of acetone solution for $2 \mathrm{~h}$. Then, $58.8 \mathrm{~g}$ of $\mathrm{EP}$ was heated for $0.5 \mathrm{~h}$ at $80{ }^{\circ} \mathrm{C}$ and then mixed into the above system with another $3 \mathrm{~h}$ of ultrasonic stirring. Afterward, the solvent was heated and continually stirred in oil bath at $80{ }^{\circ} \mathrm{C}$ for $10 \mathrm{~h} .12 .0 \mathrm{~g}$ of curing agents were melted at $100^{\circ} \mathrm{C}$ for $0.5 \mathrm{~h}$ and then added into the above system. The EP/2\% CoAlLDH@ZIF-67 sample was solidified at $100{ }^{\circ} \mathrm{C}$ for $2 \mathrm{~h}$ and then post-solidified at $150{ }^{\circ} \mathrm{C}$ for another $2 \mathrm{~h}$. After the specimen was cooled to ambient temperature, the process of preparing $\mathrm{EP} / 2 \%$ CoAl-LDH@ZIF-67 was completed. As for the preparation of EP/ $2 \%$ CoAl-LDH, and EP/2\% ZIF-67 composites, similar procedures to the above were performed. The content of the fillers in EP composites was consistent with that of $\mathrm{EP} / 2 \% \mathrm{CoAl}-$ LDH@ZIF-67.

\subsection{Characterization}

X-ray diffraction (XRD) was performed with the help of an X-ray diffractometer (Rigaku Co., Japan) complete with a Ni filter $(\lambda=$ $0.154 \mathrm{~nm}$ ) and a Cu Ka tube. Fourier transform infrared (FTIR) was conducted by using a Nicolet 6700 spectrometer (Nicolet Instrument Co.). The structures of ZIF-67, CoAl-LDH and CoAlLDH@ZIF-67 were observed by TEM (Hitachi model H-800, JEM-2011, Japan Electron Optics Laboratory). Thermogravimetric analysis (TGA) was obtained using a Q5000 thermal analyzer (TA Instruments Inc., New Castle, DE, USA) under a nitrogen flow of $25 \mathrm{~mL} \mathrm{~min}{ }^{-1}$. The fire safety of polymer materials was measured by cone calorimeter test according to ASTM E1354/ISO 5660. The morphology of the remaining char acquired from the cone test was investigated using scanning electron microscopy (SEM, Hitachi SU-8010, Japan). Raman spectroscopy was processed with a RM-1000 laser Raman 
spectrometer (Renishaw Co., UK) with excitation supplied in backscattering geometry by a $514.5 \mathrm{~nm}$ argon laser line.

\section{Results and discussion}

\subsection{Characterization of CoAl-LDH@ZIF-67 hybrids}

The crystal morphologies of ZIF-67, CoAl-LDH and CoAlLDH@ZIF-67 are characterized by XRD. The XRD patterns of these three samples are presented in Fig. 1a. The representative reflections of ZIF-67, (011), (002), (012) and (222) reflections, correspond to standard ZIF-67 structure. ${ }^{22}$ The characteristic diffraction peaks of CoAl-LDH, (003), (006), (012), and (015) reflections, are in good agreement with typical hydrotalcite-like structure (JCPDS no. 4-191). ${ }^{23}$ The XRD patterns of CoAlLDH@ZIF-67 hybrids exhibit the diffraction peaks of CoAl-LDH and ZIF-67 phases simultaneously, indicating that the successful formation of CoAl-LDH@ZIF-67 hybrids by in situ synthesis process. Moreover, it is visible that the intensity of the characteristic peaks of CoAl-LDH in the CoAl-LDH@ZIF-67 hybrids is relatively weak, in comparison with that of pristine CoAl-LDH. It can be inferred that the thick ZIF-67 layers are formed on the surface of the thin LDH nanoplates by in situ growth.

The FTIR patterns of CoAl-LDH, ZIF-67 and CoAl-LDH@ZIF-67 hybrids are shown in Fig. 1b. A clear indication of ZIF-67 peaks is primarily attributed to the ligand 2-methylimidazole. The peaks shown at 762, 1100, $1434 \mathrm{~cm}^{-1}$ are ascribed to the stretching and bending modes of the imidazole ring, while the peak at $1631 \mathrm{~cm}^{-1}$ are related to the stretching mode of $\mathrm{C}=\mathrm{N}$ bond in the ligand 2methylimidazole. The spectra also confirm the stretching mode of $\mathrm{C}-\mathrm{H}$ from the aromatic ring and the aliphatic chain in 2-methylimidazole as shown by peaks at 2931 and $2864 \mathrm{~cm}^{-1}$ respectively. ${ }^{24,25}$ In the FTIR spectra of CoAl-LDH, the broad absorption band at $3434 \mathrm{~cm}^{-1}$ is attributed to $\mathrm{O}-\mathrm{H}$ stretching of water molecule in the brucite-like layers, and the bending vibration of water molecules manifests the peak at $1631 \mathrm{~cm}^{-1}$, exhibiting the existence of water in the interlayer of $\mathrm{LDH}$. The feature of nitrates is also presented in the spectra of $\mathrm{LDH}$, authoring that nitrates are in the interlayer space. The lattice vibration peaks at 566, 632, $795 \mathrm{~cm}^{-1}$ are caused by $\mathrm{Co}-\mathrm{O}$ and $\mathrm{Al}-\mathrm{O}$ groups. ${ }^{26-28}$ In the spectrum of CoAl-LDH@ZIF-67 hybrids, it can be detected that the strength of absorption band at $1355 \mathrm{~cm}^{-1}$ has decreased obviously, compared with that of CoAl-LDH, indicating the replacement of nitrates by ZIF-67. Meanwhile, the observed peaks of CoAlLDH@ZIF-67 hybrids are similar to the peaks of ZIF-67 at corresponding position in general, demonstrating that they have similar structural information, which further confirms the fact that the LDH nanosheets are wrapped by ZIF-67 layers.

TEM results presented in Fig. 2 further reveal the microstructure of ZIF-67, CoAl-LDH and CoAl-LDH@ZIF-67. It can be distinctly found from Fig. 2a that the pristine CoAl-LDH exhibit sheet-like shape, and Fig. 2b shows that the ZIF-67 samples exhibit a hexagon shape. It can also be observed from Fig. $2 \mathrm{c}$ that the CoAl-LDH@ZIF-67 hybrids display an attractive sandwichlike structure, where the CoAl-LDH serves as a template and the ZIF-67 microscopic hexahedron grows on the surface of CoAl$\mathrm{LDH}$, which also explains the weakness of peaks of CoAl-LDH in the CoAl-LDH@ZIF-67 hybrids. The microstructures of the CoAlLDH@ZIF-67 hybrids are further characterized by SEM and elemental mapping. As shown in Fig. 3a, the SEM image clearly demonstrates that the as-synthesized CoAl-LDH@ZIF-67 hybrids have a typical sandwich-like structure, and element mapping (Fig. 3b-f) of the selected area further shows the spatial distributions of Al, O, N, C, and Co in CoAl-LDH@ZIF-67 hybrids, suggesting the presence of $\mathrm{Co}-\mathrm{Al}$ LDH and ZIF-67 particles. TEM and SEM results clearly demonstrate that the sandwich-like structured CoAl-LDH@ZIF-67 hybrids are successfully obtained.

The thermal degradation behavior of ZIF-67, CoAl-LDH and CoAl-LDH@ZIF-67 is studied by thermogravimetric analysis. The TGA and DTG curves of ZIF-67, CoAl-LDH and CoAl-LDH@ZIF-67 are depicted in Fig. 4. For ZIF-67, the pyrolysis process is divided into three phases. The first stage of weight loss is the evaporation of methanol molecules adsorbed on the surface of ZIF-67, less than $200{ }^{\circ} \mathrm{C}$. The second stage is owing to the carbonization of 2methylimidazole molecules in the pores of ZIF- 67 from $200{ }^{\circ} \mathrm{C}$ to $425{ }^{\circ} \mathrm{C}$. When the temperature reaches a certain level, the organic groups and ZIF-67 nanocrystals would break down, which just explain the final phase above $425{ }^{\circ} \mathrm{C} .{ }^{29,30}$ The TGA curve of the LDH powder exhibits two stages of mass loss at 180 and $256^{\circ} \mathrm{C}$. Evaporation of water in the hydrated phase, breakdown and carbonization of the combustible elements and dehydroxylation of the LDH, separately, may account for these mass losses. ${ }^{31}$ As for the curve of CoAl-LDH@ZIF-67 hybrids, it integrates the
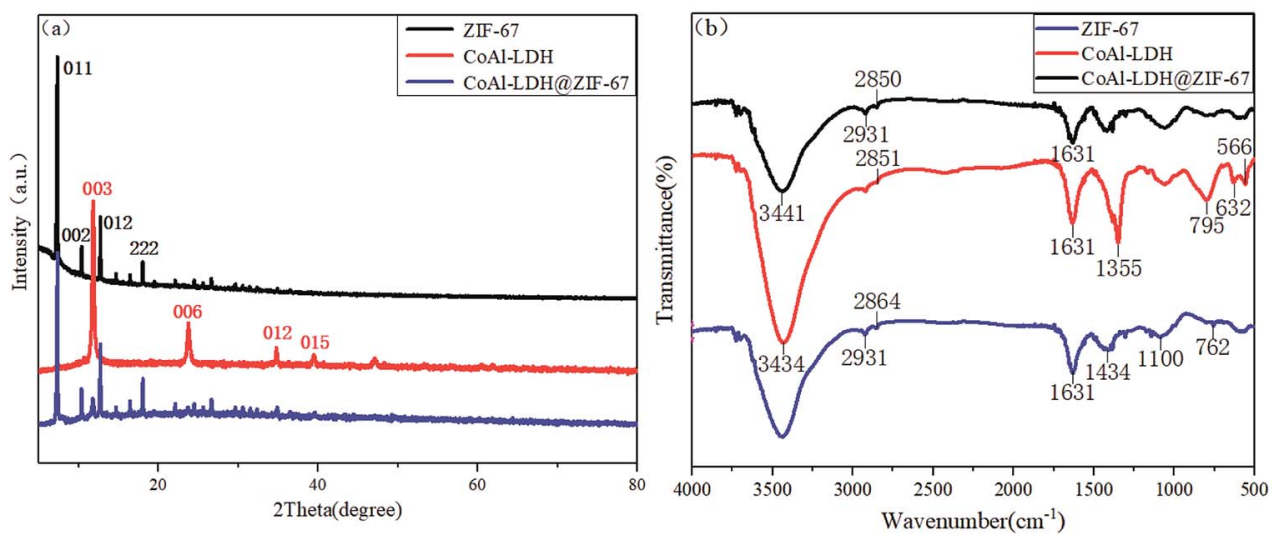

Fig. 1 XRD patterns (a) and FTIR spectra (b) of ZIF-67, CoAl-LDH and CoAl-LDH@ZIF-67. 


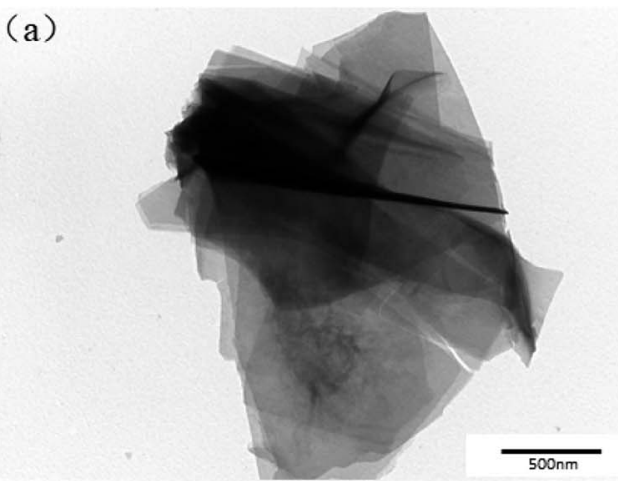

(c)

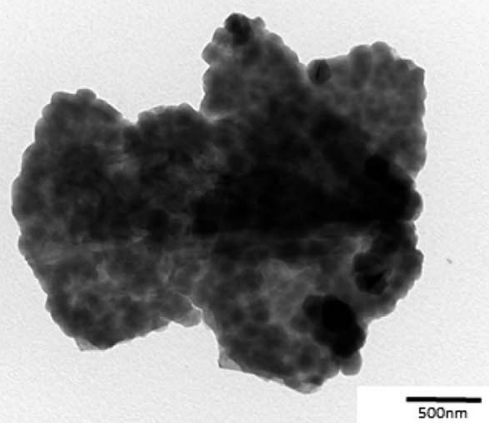

(b)
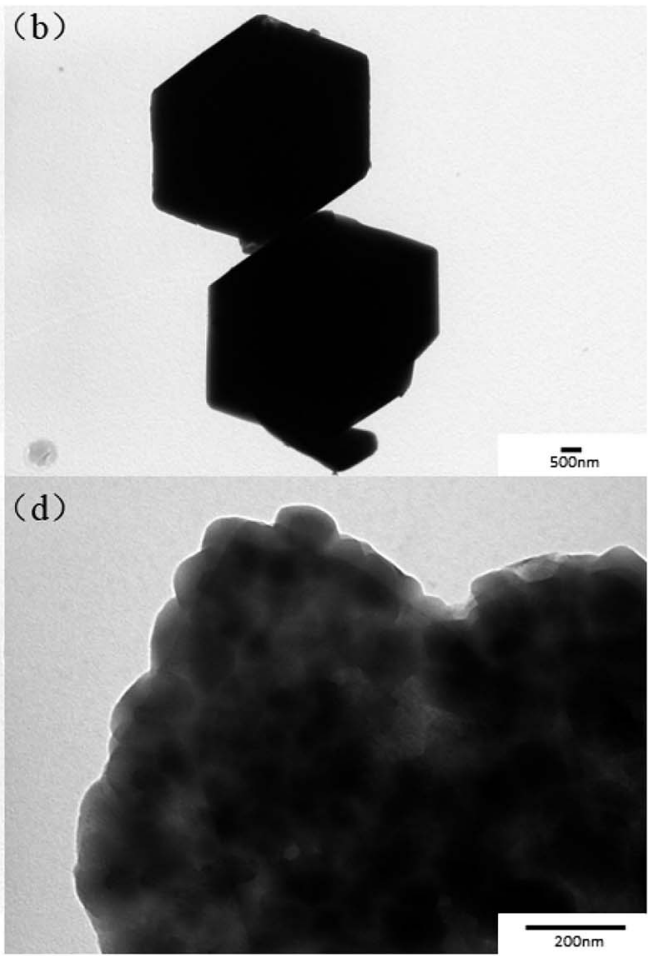

Fig. 2 TEM images of COAl-LDH (a), ZIF-67 (b) and CoAl-LDHaZIF-67 (c and d).
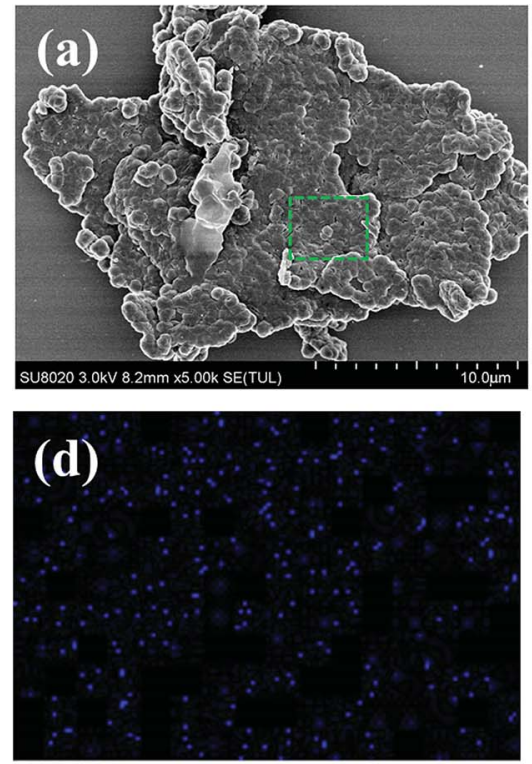

NKa1_2

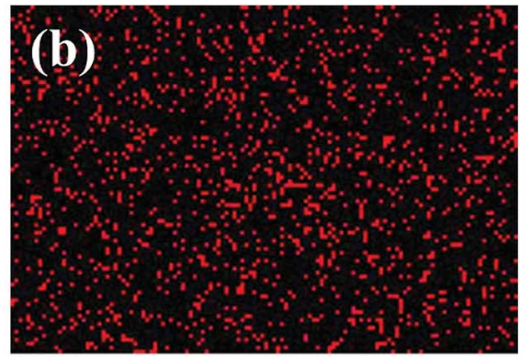

Al Ka1

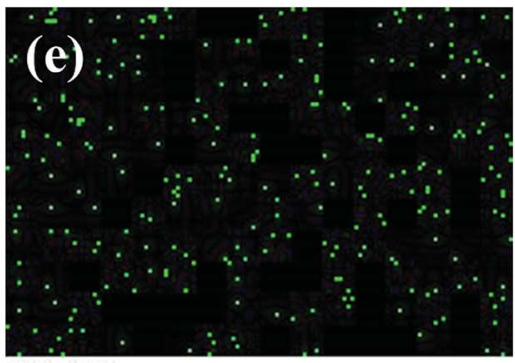

CKa1_2

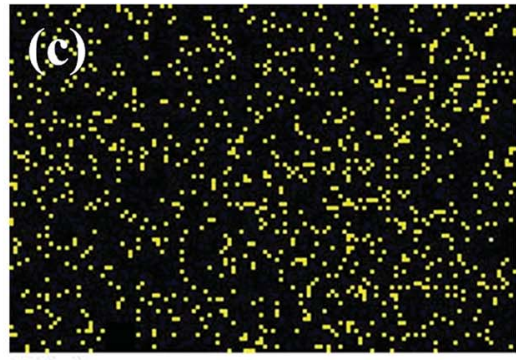

OKa1

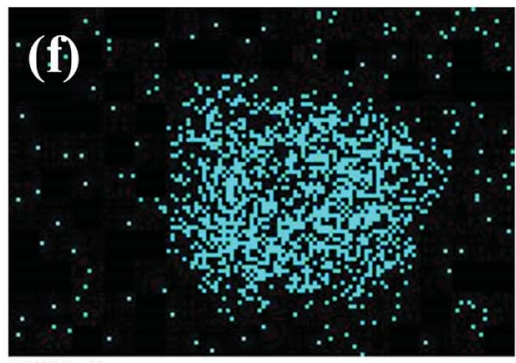

CoKa1

Fig. 3 SEM image (a) and elemental mapping (b-f) of CoAl-LDH@ZIF-67 hybrids.

features of the other two curves. The mass loss from room temperature to $425{ }^{\circ} \mathrm{C}$ is decreased, which confirms ZIF-67 successfully combined with CoAl-LDH as fillers and intensified its thermal stability.

\subsection{Thermal properties of EP composites}

The thermal degradation behavior of EP and EP composites are usually investigated by thermogravimetric analysis. The TGA and DTG curves are exhibited in Fig. 5, and some important results are shown in Table 1. $T_{10 \%}, T_{50 \%}$ and $T_{\max }$ in Table 1 stand for the temperature at $10 \mathrm{wt} \%$ weight loss, $50 \mathrm{wt} \%$ weight losses and the maximum weight loss rate, respectively. The $T_{10 \%}, T_{50 \%}$ and $T_{\max }$ of all EP composites are lower than those of pristine EP, which are possibly caused by catalytic decomposition effect of metal oxides generated from additives to prompt the pyrolysis and charring of EP at a lower temperature. ${ }^{32}$ 

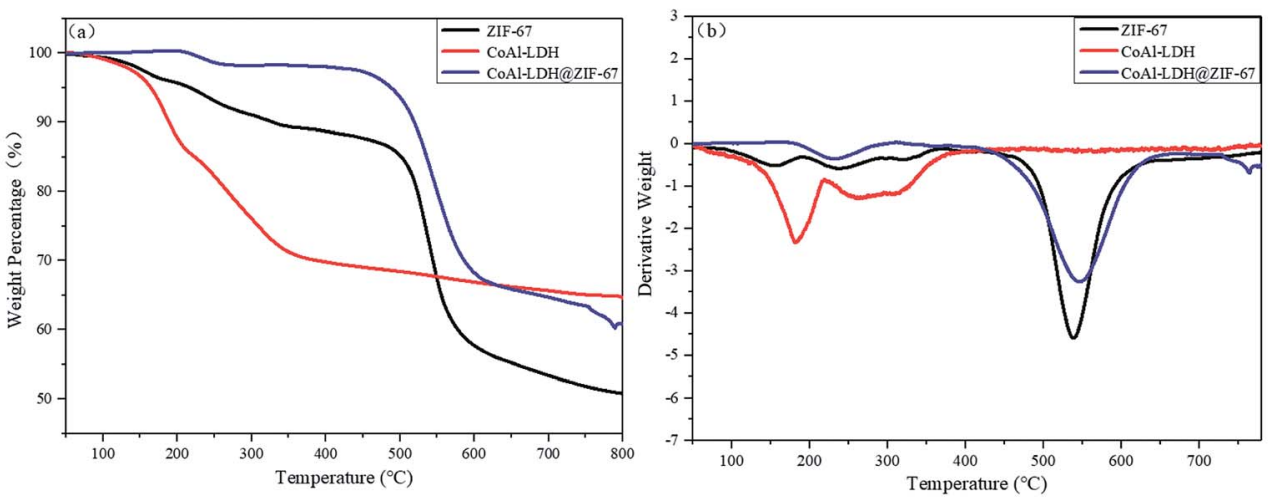

Fig. 4 TGA (a) and DTG (b) curves of ZIF-67, CoAl-LDH and CoAl-LDH@ZIF-67.
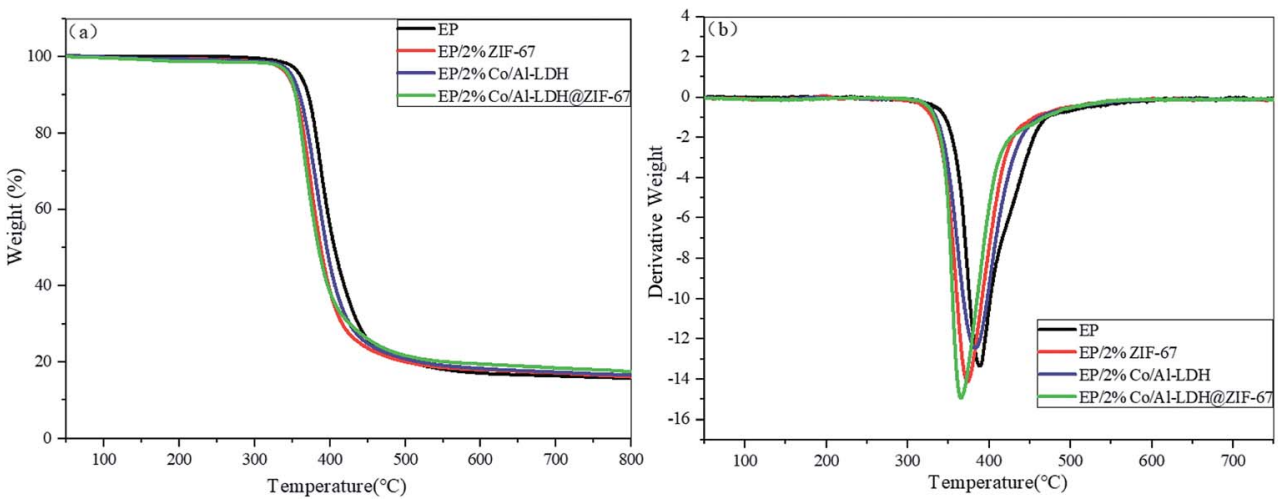

Fig. 5 TGA (a) and DTG (b) curves of neat EP and EP composites.

Table 1 TG data of neat EP and EP composites

\begin{tabular}{lcccc}
\hline & & & & \\
Sample & $T_{10 \%}\left({ }^{\circ} \mathrm{C}\right)$ & $T_{50 \%}\left({ }^{\circ} \mathrm{C}\right)$ & $T_{\max }\left({ }^{\circ} \mathrm{C}\right)$ & $\begin{array}{l}\text { Char } \\
\text { yield }(\%)\end{array}$ \\
\hline EP & 371.3 & 405.9 & 388.8 & 15.7 \\
EP/2\% ZIF-67 & 354.9 & 387.7 & 372.8 & 16.1 \\
EP/2\% CoAl-LDH & 359.8 & 395.7 & 381.8 & 16.6 \\
EP/2\% CoAl-LDH@ZIF-67 & 354.4 & 387.9 & 365.2 & 17.5
\end{tabular}

Additionally, with regard to the char production at $800{ }^{\circ} \mathrm{C}$, the mass of all EP composites char yield is more than that of pristine EP. More char residues (17.5\%) was left over in the EP composite with 2 wt\% CoAl-LDH@ZIF-67 hybrids, in comparison with EP (15.7\%), EP/2\% ZIF-67 (16.1\%) and EP/2\% CoAlLDH (16.6\%) composites. The high char residues help reduce the further degradation of products and slow down the oxygen exchange and heat and mass transfer.

\subsection{Fire performance of EP composites}

The fire properties of polymer composites are important for assessing the fire safety of materials. Cone calorimeter test is a widespread and accepted measurement method for determining the heat release rate (HRR), smoke produce rate (SPR), $\mathrm{CO}$ produce rate $(\mathrm{COP})$ and $\mathrm{CO}_{2}$ produce rate $\left(\mathrm{CO}_{2} \mathrm{P}\right)$ of materials during combustion. ${ }^{33}$ Therefore, the fire hazard of EP and
EP composites was evaluated by cone calorimeter. The HRR, SPR, COP and $\mathrm{CO}_{2} \mathrm{P}$ curves are exhibited in Fig. 6 and several significant parameters acquired from the cone calorimeter test, such as the time to ignition (TTI), total heat release (THR), average mass loss rate (AMLR) and fire growth rate index (FIGRA), are also listed in Table 2. It can be found that neat EP is highly flammable with a high PHRR of $1053 \mathrm{~kW} \mathrm{~m}^{-2}$. Compared with pristine EP, EP/2\% ZIF-67 and EP/2\% CoAl-LDH exhibit better flame retardancy, the PHRR values decrease from 1053 to 1004 and $881 \mathrm{~kW} \mathrm{~m}^{-2}$ respectively. Nevertheless, regarding EP/ 2\% CoAl-LDH@ZIF-67 composites, its PHRR value, $775 \mathrm{~kW}$ $\mathrm{m}^{-2}$, exhibits a further decrease. Besides, it can also be received from the SPR lines in Fig. 6b that EP composites show a lower SPR than neat EP, which demonstrate the additives can inhibit the material from releasing smoke. The $\mathrm{CO}$ production and $\mathrm{CO}_{2}$ production for EP and its composites are depicted in Fig. 6c and d. It can be observed that the introduction of CoAl-LDH@ZIF-67 hybrids significantly reduces $\mathrm{CO}$ production and $\mathrm{CO}_{2}$ production in comparison with pure EP, EP/2\% ZIF-67, EP/2\% CoAl$\mathrm{LDH}$ composites during combustion. The toxic CO released and $\mathrm{CO}_{2}$ produced are decreased by $59.8 \%$ and $32.2 \%$, respectively, in comparison with neat EP. Moreover, it can be discovered from Table 2 that EP composites containing the hybrids have a visible influence on the TTI. The TTIs of EP/2\% CoAlLDH@ZIF-67 is $59 \mathrm{~s}$, which need more time to ignite than pure EP. The delayed TTI suggests enhanced fire safety. As for 

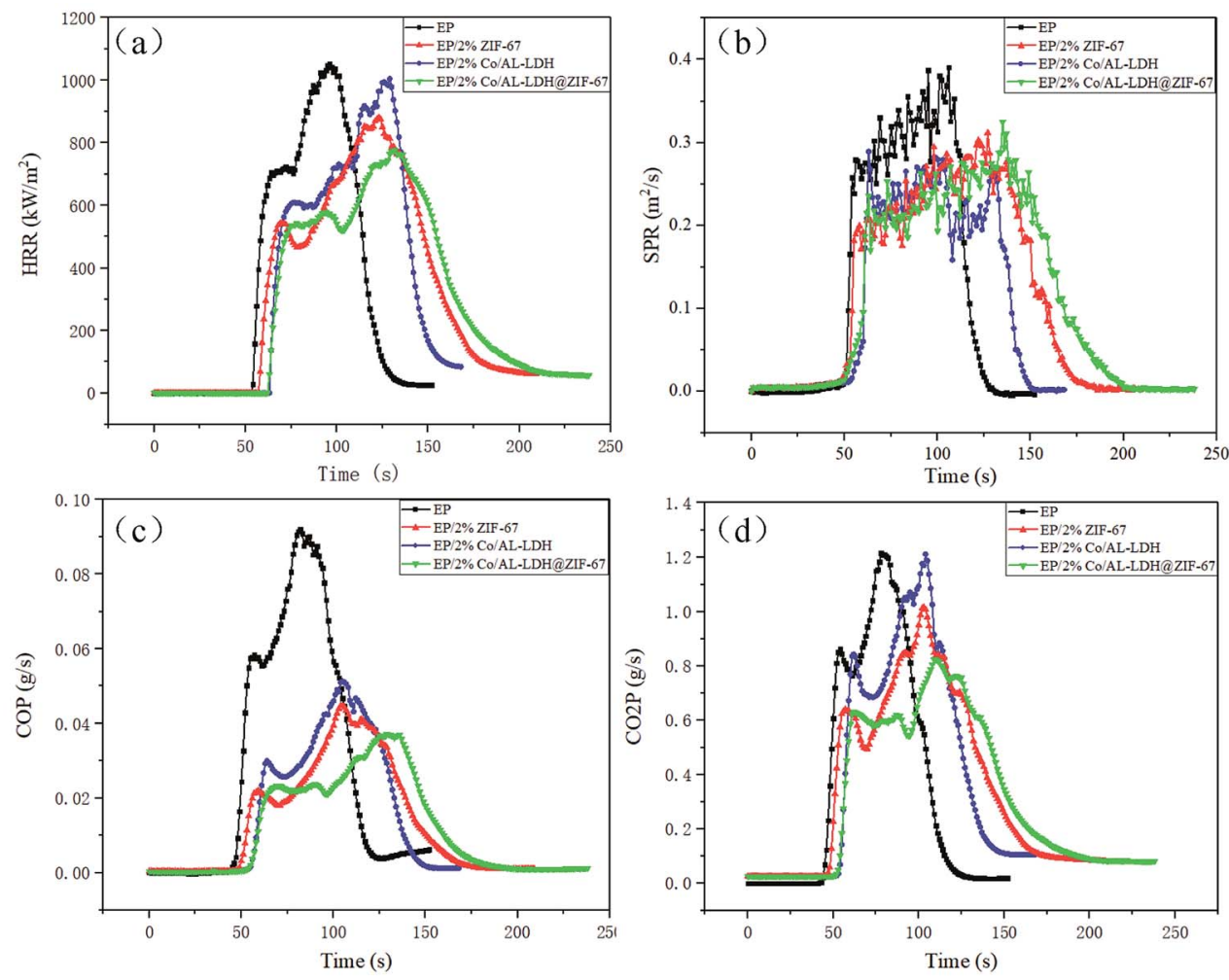

Fig. $6 \mathrm{HRR}(\mathrm{a})$, SPR (b), $\mathrm{CO}$ (c), $\mathrm{CO}_{2}$ (d) curves of neat EP and EP composites.

Table 2 Cone calorimeter data of neat EP and EP composites

\begin{tabular}{|c|c|c|c|c|c|}
\hline Sample & TTI (s) & PHRR $\left(\mathrm{kW} \mathrm{m}^{-2}\right)$ & THR $\left(\mathrm{MJ} \mathrm{m}^{-2}\right)$ & $\operatorname{AMLR}\left(\mathrm{g} \mathrm{s}^{-1}\right)$ & FIGRA $\left(\mathrm{kW} \mathrm{m}^{-2} \mathrm{~s}^{-1}\right)$ \\
\hline EP & 51 & 1053 & 90.5 & 0.255 & 11.0 \\
\hline EP/2\% ZIF-67 & 53 & 1004 & 67.3 & 0.167 & 7.8 \\
\hline EP/2\% CoAl-LDH@ZIF-67 & 59 & 775 & 67.0 & 0.139 & 5.9 \\
\hline
\end{tabular}

the AMLR, all composites show lower AMLR in comparison to pure EP. The THR values of EP composites reduce at different extent, in comparison to that of neat EP. The FIGRA is usually used to characterize the rate of fire growth. The larger the FIGRA index, the faster the combustion growth, which means the higher fire risk. It can be obtained by dividing the value of PHRR by the time to PHRR. The FIGRA value of pure EP is $11.0 \mathrm{~kW} \mathrm{~m}^{-2}$ $\mathrm{s}^{-1}$, while those of EP/2\% ZIF- 67 and EP/2\% CoAl-LDH composites are 7.8 and $7.2 \mathrm{~kW} \mathrm{~m}^{-2} \mathrm{~s}^{-1}$. The FIGRA value of $\mathrm{EP} / 2 \%$ CoAl-LDH@ZIF-67 composites is $5.9 \mathrm{~kW} \mathrm{~m}^{-2} \mathrm{~s}^{-1}$, indicating the lowest fire hazards. The improvement of the fire safety is mainly caused by physical blocking effect, catalytic carbonization of CoAl-LDH@ZIF-67 hybrids and combined action of LDH and ZIF-67.

\subsection{Analysis of char residues}

The char residue of EP and EP composites is studied by SEM after cone calorimeter test to further reveal the mechanism of flame retardant and smoke suppression. The micromorphology of the char residues of EP and EP composites is shown in Fig. 7.
Neat EP (Fig. 7a) shows a discontinuous char layer with obvious cracks and holes. However, the surfaces of char residue of EP composites (Fig. 7b-d) exhibit more continuous and dense structure, especially for EP/2\% CoAl-LDH@ZIF-67 sample. The dense and continuous surface can efficiently prevent the exchange of heat and gas between gas phase and condensed phase. Fig. $7 d$ also exhibits that the surface of $\mathrm{EP} / 2 \% \mathrm{CoAl}-$ LDH@ZIF-67 char residue is covered by a lot of granular materials, which bring a favorable physical blocking effect. In conclusion, the incorporation of CoAl-LDH@ZIF-67 hybrids can help EP to form compact carbonaceous char and effectively improve the fire safety of EP.

Laser Raman spectroscopy is a significant way to investigate the performance of char residues generated in the burning of polymer materials. Fig. 8 represents the Raman spectra of the char residues of neat EP and EP composites. All samples show two typical absorption peaks at 1604 and $1348 \mathrm{~cm}^{-1}$, which correspond to $\mathrm{G}$ and $\mathrm{D}$ peaks, respectively. The area size ratio of the $\mathrm{D}$ to $\mathrm{G}$ bands $\left(I_{\mathrm{D}} / I_{\mathrm{G}}\right)$ represents the degree of graphitization of the char residues. In principle, the higher the ratio of $I_{\mathrm{D}} / I_{\mathrm{G}}$, the lower the graphitization degree of char residue is. ${ }^{34}$ As can 

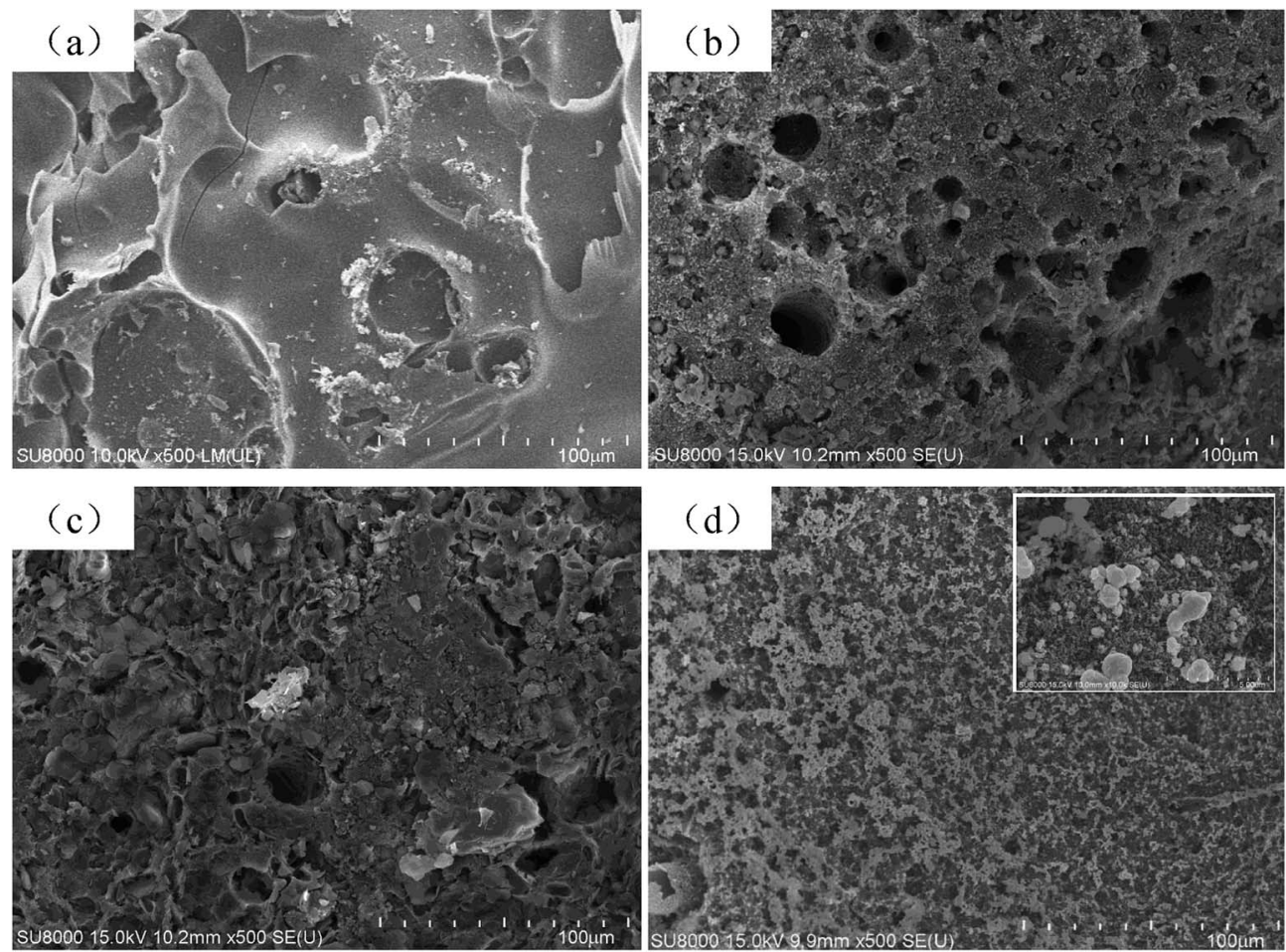

Fig. 7 SEM images of char residue of neat EP (a), EP/2\% ZIF-67 (b), EP/2\% CoAl-LDH (c), EP/2\% CoAl-LDH@ZIF-67 (d).

be seen in Fig. 8, the $I_{\mathrm{D}} / I_{\mathrm{G}}$ values follow the order of EP (2.63)> $\mathrm{EP} / 2 \%$ CoAl-LDH $(2.61)>\mathrm{EP} / 2 \%$ ZIF-67 (2.45) $>\mathrm{EP} / 2 \%$ CoAlLDH@ZIF-67 (2.31), indicating the highest graphitized residual char of the EP/2\% CoAl-LDH@ZIF-67 composites.
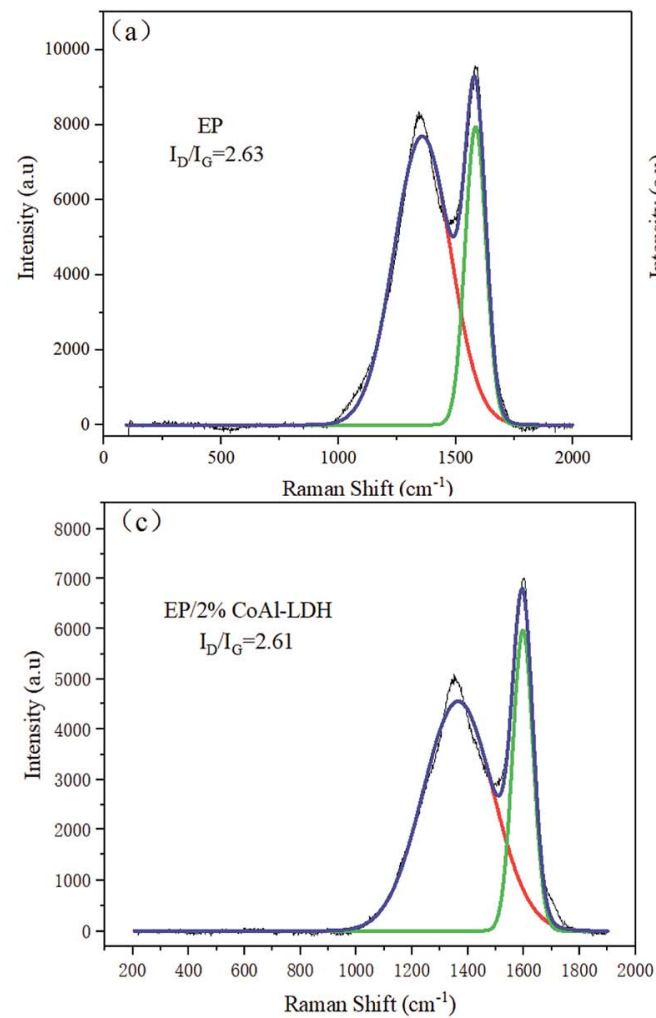

From the current results, it can be concluded that graphitized carbon is produced in the process of burning owing to the catalytic action of LDH and MOF. The char layer with high content of graphitized carbons can serve as a barrier to block
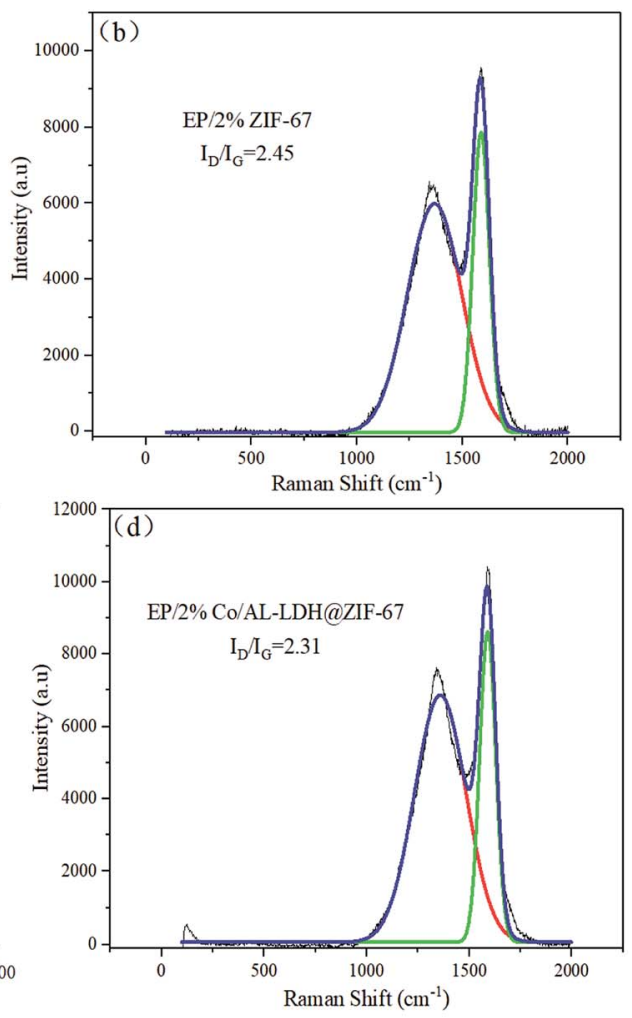

Fig. 8 Raman spectra of char residue of neat EP (a), EP/2\% ZIF-67 (b), EP/2\% CoAl-LDH (c), EP/2\% CoAl-LDH@ZIF-67 (d). 

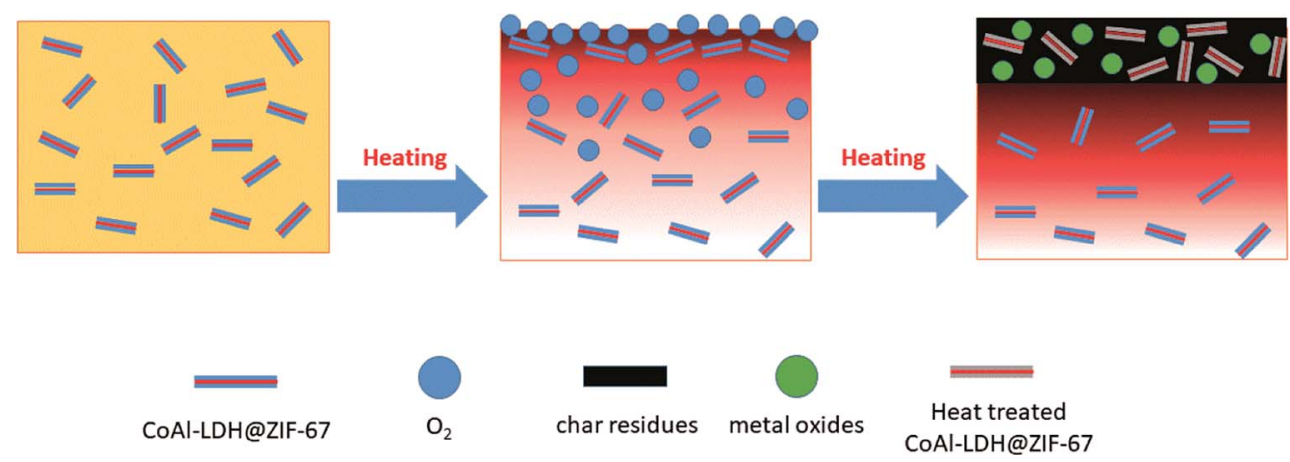

Scheme 1 Schematic illustration for flame retardant mechanism of EP/2\% CoAl-LDH@ZIF-67 composites.

the exchange of mass and heat between the gas phase and the condensed phase, inhibit the organic volatiles from volatilizing, and reduce the heat release rate in burning process. ${ }^{35}$

Based on above research, the possible mechanism of flame retardancy and smoke suppression performances of the $\mathrm{EP} / 2 \%$ CoAl-LDH@ZIF-67 composites can be inferred and depicted in Scheme 1. Three possible reasons for improving fire safety of EP composites by incorporating CoAl-LDH@ZIF-67 hybrids are proposed. Firstly, the sandwich-like structure of CoAlLDH@ZIF-67 hybrids acts as a physical barrier in the early stage of pyrolysis, which can slow down the transfer of heat and oxygen and prevent further reaction with degradable products. Secondly, the thermal degradation of CoAl-LDH@ZIF-67 hybrids is an endothermic process, which would decrease the system temperature and contribute to the improvement the fire safety. Finally, metal oxides generated from CoAl-LDH@ZIF-67 hybrids in the burning process promote the production of char residue and form a more compact char layer.

\section{Conclusion}

In present study, sandwich-like structured CoAl-LDH@ZIF-67 hybrids were successfully synthesized, and its structure and morphology were determined by XRD, FTIR, TEM and SEM. The morphology study shows that ZIF-67 nanoparticles were decorated on the surface of CoAl-LDH nanoplates. Subsequently, CoAl-LDH@ZIF-67 hybrids were used for improving the thermal and fire safety of EP composites. TGA results show the $T_{10 \%}$, $T_{50 \%}$ and $T_{\max }$ value of EP composites with CoAl-LDH@ZIF-67 hybrids are decreased to some extent, but the char yield is higher than that of pristine EP. The values of PHRR, COP and $\mathrm{CO}_{2} \mathrm{P}$ of EP/2\% CoAl-LDH@ZIF-67 are the lowest in all EP composites. In addition, other important parameters obtained from the cone tests shows EP/2\% CoAl-LDH@ZIF-67 have excellent fire safety. The obvious improvements of these properties are primarily assigned to the physical barrier effect and endothermic effect of CoAl-LDH@ZIF-67 hybrids, catalytic carbonization effect of metal oxides generated from CoAlLDH@ZIF-67 and combination effect between LDH and MOF.

\section{Conflicts of interest}

There are no conflicts to declare.

\section{Acknowledgements}

This work was supported by the Fundamental Research Funds for the Central Universities, China University of Geosciences (Wuhan) (CUG160607), Natural Science Fund of Hubei Province (No. 2017CFB315), Opening Project of Engineering Research Center of Rock-Soil Drilling \& Excavation and Protection, (China University of Geosciences (Wuhan)), Ministry of Education (201801), Scientific Research Plan Guidance Project of Hubei Province (B2017594).

\section{References}

1 Y. Zhou, J. Feng, H. Peng, H. Qu and J. Hao, Polym. Degrad. Stab., 2014, 110, 395-404.

2 K. Q. Zhou, C. K. Liu and R. Gao, Composites, Part A, 2018, 112, 432-443.

3 J. H. Zhang, Q. H. Kong and D. Y. Wang, J. Mater. Chem. A, 2018, 6, 6376-6386.

4 G. Huang, S. Chen, P. Song, P. Lu, C. Wu and H. Liang, Appl. Clay Sci., 2014, 78-85.

5 P. Ding, B. Kang, J. Zhang, J. Yang, N. Song, S. Tang and L. Shi, J. Colloid Interface Sci., 2015, 440, 46-52.

6 L. Qiu, W. Chen and B. Qu, Polym. Degrad. Stab., 2005, 87, 433-440.

7 S. Yang, J. Wang, S. Huo, J. Wang and Y. Tang, Polym. Degrad. Stab., 2016, 126, 9-16.

8 F. R. Costa, U. Wagenknecht and G. Heinrich, Polym. Degrad. Stab., 2007, 92, 1813-1823.

9 W. Xu, B. Zhang, X. Wang, G. Wang and D. Ding, J. Hazard. Mater., 2017, 343, 364-375.

10 X. Wang, S. Zhou, W. Xing, B. Yu, X. Feng, L. Song and Y. Hu, J. Mater. Chem. A, 2013, 1, 4383-4390.

11 E. N. Kalali, X. Wang and D. Y. Wang, J. Mater. Chem. A, 2015, 3, 6819-6826.

12 S. Song, J. Ma, K. Cao, G. Chang, Y. Huang and J. Yang, Polym. Degrad. Stab., 2014, 99, 43-52.

13 S. Carlino, Solid State Ionics, 1997, 98, 73-84.

14 Z. Wang, E. Han and W. Ke, Prog. Org. Coat., 2005, 53, 29-37. 15 Y. Liu, N. Wang, J. H. Pan, F. Steinbach and J. Caro, J. Am. Chem. Soc., 2014, 136, 14353-14356.

16 S. Keskin and D. S. Sholl, Energy Environ. Sci., 2010, 3, 343351. 
17 X. W. Shi, X. Dai, Y. Cao, J. W. Li, C. G. Huo and X. L. Wang, Ind. Eng. Chem. Res., 2017, 56, 3887-3894.

18 Y. B. Hou, W. Z. Hu, Z. Gui and Y. Hu, Compos. Sci. Technol., 2017, 152, 231-242.

19 P. F. Liu, K. Tao, G. C. Li, M. K. Wu, S. R. Zhu, F. Y. Yi, W. N. Zhao and L. Han, Dalton Trans., 2016, 45, 1263212635.

20 Z. H. Li, M. F. Shao, L. Zhou, R. K. Zhang, C. Zhang, M. Wei, D. G. Evans and X. Duan, Adv. Mater., 2016, 28, 2337-2344.

21 J. B. Han, J. Lu, M. Wei, Z. L. Wang and X. Duan, Chem. Commun., 2008, 44, 5188-5190.

22 Y. Dou, J. Zhou, F. Yang, M. Zhao, Z. R. Nie and J. R. J. Li, J. Mater. Chem. A, 2016, 4, 12526-12534.

23 A. Garciagallastegui, D. Iruretagoyena, V. Gouvea, M. Mokhtar, A. M. Asiri, S. N. Basahel, S. A. Althabaiti, A. O. Alyoubi, D. Chadwick and M. S. P. Shaffer, Chem. Mater., 2012, 24, 4531-4539.

24 A. F. Gross, E. Sherman and J. J. Vajo, Dalton Trans., 2012, 41, 5458-5460.

25 K. Y. A. Lin and H. A. Chang, J. Taiwan Inst. Chem. Eng., 2015, 53, 40-45.
26 L. Zhang, X. Zhang, L. Shen, B. Gao, L. Hao, X. Lu, F. Zhang, B. Ding and C. Yuan, J. Power Sources, 2012, 199, 395-401.

27 Z. A. Hu, Y. L. Xie, Y. X. Wang, H. Y. Wu, Y. Y. Yang and Z. Y. Zhang, Electrochim. Acta, 2009, 54, 2737-2741.

28 L. Yan, H. Kong and Z. J. Li, Acta Chim. Sin., 2013, 71, 822.

29 X. Yan, Y. Yang, X. Hu, M. Zhou and S. Komarneni, Microporous Mesoporous Mater., 2016, 234, 162-165.

30 A. Schejn, L. Balan, V. Falk, L. Aranda, G. Medjahdi and R. Schneider, CrystEngComm, 2014, 16, 4493-4500.

31 E. Conterosito, L. Palin, D. Antonioli, D. Viterbo, E. Mugnaioli, U. Kolb, L. Perioli, M. Milanesio and V. Gianotti, Chem.-Eur. J., 2015, 21, 14975-14986.

32 K. Q. Zhou, R. Gao and X. D. Qian, J. Hazard. Mater., 2017, 338, 343-355.

33 K. Q. Zhou, G. Tang, R. Gao and S. D. Jiang, J. Hazard. Mater., 2018, 344, 1078-1089.

34 W. Y. Xing, W. Yang, W. J. Yang, Q. H. Hu, J. Y. Si, H. D. Lu, B. H. Yang, L. Song, Y. Hu and R. K. K. Yuen, ACS Appl. Mater. Interfaces, 2016, 8, 26266-26274.

35 K. Q. Zhou, G. Tang, R. Gao and H. L. Guo, Composites, Part A, 2018, 107, 144-154. 